\title{
¿Qué comemos? Análisis de la relación entre condiciones socioeconómicas y productos alimentarios de los hogares colombianos
}

\author{
What do we eat? \\ Socio-economic conditions and households \\ food consumption in Colombia
}

\begin{abstract}
Giselle Torres-Pabón ${ }^{1}$
Forma de citar: Torres Pabón G. ¿Qué comemos? Análisis de la relación entre condiciones socioeconómicas y productos alimentarios de los hogares colombianos. Salud UIS. 52(3): 251-260. doi: http:/dx.doi.org/10.18273/revsal.v52n3-2020007 (c) (1)
\end{abstract}

\section{Resumen}

Introducción: los hogares consumen (o no) los alimentos que sus condiciones socioeconómicas les permiten (o no). Objetivo: analizar los productos que consumen los hogares colombianos a partir de sus condiciones socioeconómicas. Metodología: 1. Análisis descriptivo y 2. Análisis de Cluster, enlace Ward. Se utilizó la encuesta de Presupuestos de los Hogares 2016-2017, realizada por el Departamento Nacional de Estadística colombiano. Resultados: se evidencian dos grandes grupos de tipologías de hogares: aquellos que consumen alimentos principalmente por fuera del hogar o productos ya preparados; y aquellos hogares que distribuyen su presupuesto del hogar en alimentos para preparar. Y dentro de aquellos hogares que gastan su presupuesto alimentario en alimentos para preparar, se encontraron tres principales grupos. Conclusiones: los resultados demuestran estratificación social en las prácticas alimentarias de los hogares colombianos.

Palabras clave: Ingestión de alimentos; Alimentos; Clase social; Vivienda; Presupuestos; Análisis por conglomerados; Obesidad; Política pública.

\footnotetext{
Abstract

Introduction: Households consume (or not) the food that their socio-economic conditions allow them (or not). Objective: To analyze the products that Colombian households consume based on their socio-economic conditions. Methodology: 1. Descriptive analysis, and 2. Cluster analysis, Ward link. I worked on the Households Budgets survey (Encuesta Nacional de Presupuesto de los Hogares 2016-2017). The Colombian National Department of

1. Pontificia Universidad Católica de Chile. Santiago de Chile

Correspondencia: Giselle Torres Pabón. Dirección: Instituto de Sociología Pontificia Universidad Católica de Chile: Avenida Vicuña Mackenna 4860, Casilla 306, Correo 22, Macul, Santiago Teléfono: +5622354 4651 Correo electrónico: gtorres1@uc.cl 
Statistics conducted the survey. Results: Two large groups of household typologies are evident: those that consume food mainly outside the home and those households that spend their household budget on food to eat at home. Conclusions: Results show social stratification in food consumption of Colombian households.

Keywords: Eating; Food; Social class; Household; Budgets; Cluster analysis; Obesity; Public policy.

\section{Introducción}

Los productos que consumen los hogares son diferentes dadas sus características sociales y económicas ${ }^{1-3}$. El análisis de la relación entre productos alimentarios y condiciones socioeconómicas de los hogares tiene al menos dos principales vertientes: una médica o funcional y otra sociológica. En perspectiva médica, Darmon y Drewnoski ${ }^{4}$ hallaron que dietas densas energéticamente y bajas en nutrientes son consumidas principalmente por personas con bajos niveles socioeconómicos. Y, en general, se ha encontrado que a mayores niveles socioeconómicos más sanos son los patrones de las dietas ingeridas ${ }^{5-9}$. Entre los productos asociados a los niveles socioeconómicos altos están: frutas, verduras, queso, leche, pescado ${ }^{5-9}$, mientras que entre los niveles socioeconómicos más bajos se pueden encontrar los productos grasos y los dulces ${ }^{5-9}$.

En perspectiva funcional, Marvin Harris ${ }^{10}$ plantea que los hogares consumen los alimentos que les reportan mayores beneficios en tiempo de obtención, precios o nutrientes. Lo que implica, necesariamente, un cálculo utilidad/beneficios/costos. Pero en sociología, especialmente en la Sociología Económica, no se concibe que en todas las acciones humanas subyace un cálculo $^{11}$; se considera que hay otros elementos — como las formas de comprender al mundo, las cosas y sus usos, los conocimientos previos de comprensión, el saberhacer, los estados emocionales, las motivacionesque afectan la práctica social ${ }^{12,13} \mathrm{y}$ el sentido de la acción $^{14}$. Por lo tanto, en perspectiva sociológica, no sólo los ingresos o los cálculos costo/precio/beneficio definen las elecciones o los gustos alimentarios. Los usos alimentarios —esto es: definir qué comer, dónde comprar, cuándo, cuánto, con quién- no es una actividad sencilla o simple, dado que se requiere de un gran conjunto de dispositivos para hacerlo posible, y no es sólo una cuestión de oferta y demanda de un bien o servicio $^{15-17}$.

En sociología un autor clásico en el análisis de las diferencias sociales por medio de bienes de consumo, como los alimentos, es Pierre Bourdieu. En lo que respecta al consumo de alimentos, el autor considera que el habitus moldea el consumo. En ese sentido, las clases altas consumen lo 'fino-magro-refinado-ligero', lo 'exótico-rebuscado-sano-natural-azucarado' y bienes de 'lujo', mientras que las clases populares consumen lo 'fuerte-graso-salado-rico' y bienes de 'necesidad'1. Así, los productos de las clases altas serían: el pescado, las frutas o las verduras. Mientras que en las clases bajas se encontrarían: el cerdo, los dulces, las harinas o los productos alimentarios de consumo masivo ${ }^{1}$.

A partir de dichas clasificaciones distintivas y de los productos que corresponden a cada una, podría decirse que se construye una línea bourdiana alrededor del análisis del consumo de alimentos. Kamphuis, et al. ${ }^{18}$ mediante una revisión sistemática exponen los diferentes trabajos que se han dedicado a analizar la relación entre el concepto de capital cultural, desarrollado por Bourdieu, y elecciones alimenticias. En la revisión se pone en evidencia un consenso en los resultados obtenidos: hay una asociación positiva entre posición socioeconómica y elecciones alimenticias saludables ${ }^{18}$.

Por su parte, Wills, et al. ${ }^{19}$, también en perspectiva bourdiana, tienen como punto de partida que el consumo de alimentos es una práctica construida socialmente; y dicha práctica — que se compone de comprar, almacenar, cocinar y consumir - está constreñida por los contextos socioculturales en los que las personas existen o han existido. Mediante un análisis cualitativo, basado en el contexto sociocultural familiar cotidiano, las autoras buscan comprender las prácticas alimentarias desplegadas por los padres y jóvenes adolescentes en Escocia. Dichos contextos son definidos analíticamente por clase: baja y media. Y de acuerdo con la clase, las autoras identifican determinados patrones familiares. Para la clase baja se encuentran: limitaciones monetarias, productos congelados y pre-preparados; y respecto a los adolescentes los adultos esperan que sean responsables de sus decisiones alimentarias y tomen decisiones autónomas. Mientras que para el caso de la clase media se encuentran 'reglas' de consumo en el hogar para los adolescentes: vegetales son un consumo obligatorio y en el hogar hay una variada gama de productos: cordero, cerdo asado, pescado, entre otros. Y también se destina tiempo para su preparación y el consumo de estos en familia y en el hogar. De modo que la estructura familiar y la transmisión de prácticas 
alimentarias inciden en los patrones en corto, mediano o largo tiempo.

También, Beagan, et al. ${ }^{20}$ analizan desde la perspectiva bourdiana la compra de alimentos en las familias canadienses de bajos y altos ingresos. Los autores ya no se enfocan en los productos. Se concentran en las compras: lugares de compra y sus diferencias en precios, productos, calidad, proximidad y ofertas. Como también en las limitaciones o posibilidades propias de los hogares: presupuestos, conocimientos nutricionales o uso del tiempo.

Los resultados señalan limitaciones presupuestarias para los grupos socioeconómicos más bajos, que en la lógica de Bourdieu significa el desarrollo de gustos de necesidad. Y dichas limitaciones derivan en la movilización de diferentes habilidades, motivaciones y conocimientos para las compras alimentarias: descuentos, lugares con bajos precios, productos sin marca y control de compras impulsivas. Mientras que los grupos socioeconómicos altos se distancian de los gustos de necesidad y las compras tienden a ser actividades 'divertidas', en las que la 'calidad' y la 'autenticidad' juegan un rol principal.

Finalmente, en perspectiva bourdiana en Canadá, Bauman, et al. ${ }^{21}$ tratan de comprender las preferencias alimentarias de los grupos socioeconómicos más bajos. Se encontró que las preferencias alimentarias de los grupos socioeconómicos más bajos son modeladas por las limitaciones socioeconómicas cotidianas, lo que develó tres patrones de consumo de 'necesidad': 1. gustos de abundancia: cuya preocupación radica en la cantidad, tamaño; 2. gustos por alimentos de marcas corporativas o de consumo masivo: no tanto porque sea lo que los niveles socioeconómicos más bajos pueden pagar, sino que también lo perciben de sabor agradable; 3. Comidas 'étnicas': como oportunidades para la variedad (ej. comida china). Mientras que entre los grupos socioeconómicos más altos se identifican con consumo de alimentos naturales u orgánicos, ensaladas o vegetales.

De modo que la literatura desarrollada en Europa y otros países desarrollados como Canadá permite afirmar que hay resultados teóricos y empíricos para comprender las diferencias sociales y económicas a través de la alimentación ${ }^{22,23}$. Y dicha literatura ofrece ejes analíticos para comprender los patrones alimentarios característicos de los grupos socioeconómicos altos, medios o bajos, ya fuera por tipo de producto, cantidad, tamaño, calidad, marca u otras especificaciones.
Sin embargo, la pregunta que surge es: ¿qué ocurre en América Latina? ¿qué es (o no) aplicable de estos entramados teóricos y empíricos para los casos latinoamericanos? Algunos estudios revelan que existen diferencias en los alimentos consumidos en los hogares, dadas sus características socioeconómicas, pero los entramados empíricos varían ${ }^{24-28}$.

Primero, por ejemplo, en México, Garza-Montoya y Ramos-Tovar ${ }^{25}$, si bien no analizaron la relación entre condiciones socioeconómicas y consumo de alimentos en los hogares mexicanos, revisaron el patrón de gasto en alimentos y bebidas en los hogares mexicanos entre 1984 y 2014. Metodológicamente las autoras midieron en conjunto gasto para el consumo en alimentos, bebidas y tabaco para medir las proporciones de gasto en alimentos y reflexiones de la Encuesta de Ingresos y Gastos de los Hogares. Sin embargo, en la literatura sobre el gasto en consumo de alimentos ${ }^{16,17}$ se consideran, especialmente, las bebidas alcohólicas y el tabaco, como productos de naturaleza distinta a los productos alimentarios, en especial para crear las proporciones de gasto alimentario. A pesar de dicha falencia, dicho artículo se entenderá aquí como la presentación de los gastos de diferentes bienes de consumo de los hogares incluidos los alimentos. Las autoras encontraron el incremento de consumo de alimentos por fuera del hogar y preparados (tacos, pizza, birria, barbacoa, atoles, sopas) y de bebidas no alcohólicas. Mientras que hubo una disminución del consumo de verduras, legumbres, frutas, entre otros productos. Y el consumo de carnes en general fue alto durante el periodo.

Ahora revisando la relación consumo de alimentos y condiciones socioeconómicas en Argentina, Rodríguez et al. ${ }^{26}$, a partir de la Encuesta Nacional de Gasto de los Hogares, encontraron que las carnes rojas están presentes en la dieta de la gran mayoría de los hogares argentinos posiblemente, según los autores, por las ventajas productivas del país respecto a dicho producto, y encontraron que los hogares más carnívoros son aquellos de menor nivel de educación e ingreso ${ }^{26}$.

También en Argentina, Zapata, et al. ${ }^{28}$ encontraron que los hogares argentinos consumen mayor cantidad de gramos al día de: panes de panadería, leche, papa, carne vacuna y yerba mate. Aunque también, en perspectiva temporal, hallaron una disminución en el consumo de frutas, vegetales, legumbres y carne vacuna; y un incremento en consumo de masas de tartas y empanadas, carne porcina, productos cárnicos semielaborados y alimentos listos para consumir. Pero más aún, en términos de diferenciación socioeconómica, Bertollo 
et al. $^{24}$ reportaron que el aumento de consumo de pastas frescas y masas de tarta, empanadas y canelones aumenta conforme incrementan los ingresos del hogar; como también ocurre con los amasados de pastelería, masas para pizza y galletas (dulces y saladas). Estos dos últimos resultados son contradictorios con la literatura europea ${ }^{1,4,6}$.

De modo que de la literatura revisada se pueden extraer tres conclusiones principales para este artículo: 1 . Existen diferencias entre los productos alimentarios que consumen los hogares dadas sus características socioeconómicas, 2. Los productos alimentarios consumidos en los hogares varían según los países; y 3. Los productos alimentarios característicos de los niveles socioeconómicos altos o bajos varían según el país o el contexto en el que toma existencia la práctica alimentaria.

Así pues, el objetivo de este artículo es analizar los productos que consumen los hogares colombianos, a partir de sus condiciones sociales y económicas. La metodología utilizada es cuantitativa: 1. Análisis descriptivo y 2. Análisis de Clusters, enlace Ward. La hipótesis que guía este artículo es: los productos alimenticios que consumen los hogares son diferentes dadas sus características socioeconómicas.

Tratar de encontrar una tipología y los alimentos que reflejen la diferenciación social en Colombia es algo que todavía no se había realizado. Por lo que este trabajo pretende realizar una contribución a la literatura del consumo de alimentos y al debate que busca analizar las diferenciaciones en el consumo de alimentos, pero esta vez desde América Latina y a partir del estudio del caso colombiano.

\section{Metodología}

En este artículo se utilizó la Encuesta Nacional de Presupuesto de los Hogares (ENPH) realizada entre julio 2016 y julio 2017 en Colombia. La ENPH es representativa a nivel nacional. Las unidades de análisis son los hogares y las personas ${ }^{1}$. La encuesta tiene información sobre las características socioeconómicas de los hogares y las personas (composición del hogar, características de la vivienda, niveles educativos, sexo, entre otros) y los gastos e ingresos de ellos.

1 Se le invita al lector revisar los materiales metodológicos para verificar unidades de análisis e indicaciones de manipulación de datos: http://microdatos.dane.gov.co/index.php/catalog/566/related materials
De los gastos en alimentos se tiene información de los productos alimentarios, frecuencia, cantidades, precios y lugares de obtención. La encuesta trabajada se compone de 87.201 observaciones (hogares). Pero dado que el programa estadístico stata no procesa dicha cantidad de observaciones para el análisis de cluster, con el mismo programa se seleccionó aleatoriamente la cantidad máxima que el programa podía procesar: 8.644 observaciones (hogares).

Las variables dependientes son las proporciones de gasto por grupos de productos para cada hogar. Para hallar el gasto en cada grupo de producto se siguió la mensualización dada por el Departamento Nacional de Estadística en la ficha metodológica para las zonas urbanas y rurales. Luego, para obtener los gastos totales en alimentos de los hogares, se hizo la sumatoria de todos los grupos de productos. Con ambos cálculos se calcularon las proporciones: gasto del grupo de productos alimentarios dividido por el gasto total en alimentos, multiplicado por cien. Por lo tanto, los resultados se presentan en porcentaje. El listado de productos por los grupos de la encuesta es:

1. Pan, arepas, bollos y almojábanas

2. Galletas de sal y de dulce

3. Arroz, pastas alimenticias, avena, maíz, harinas y otros cereales

4. Carne de res, cerdo, hueso y vísceras

5. Pollo, otras aves y menudencias

6. Salchichas, jamón, mortadela, salchichón y otras carnes frías preparadas

7. Pescado de río, mar y otros productos marinos (frescos, congelados y enlatados)

8. Leche (animal y vegetal)

9. Queso (animal y vegetal)

10. Otros derivados de la leche: yogur, kumis, etc.

11. Huevos

12. Aceites y grasas: aceite, manteca, mantequilla, margarina, etc.

13. Frutas: manzanas, peras, naranjas, etc.

14. Verduras y hortalizas: tomate, cebolla, arveja, espinaca, apio, zanahoria, etc.

15. Granos secos: fríjol, lenteja, garbanzo, etc.

16. Enlatados y encurtidos: arveja, fríjol, maíz, espárragos, zanahoria, habichuela, etc.

17. Plátano, yuca, arracacha, ñame, papa y otros tubérculos

18. Azúcar, panela y otros endulzantes

19. Mermeladas, arequipe, bocadillos, compotas, dulces, helados, etc.

20. Salsas, mayonesa, mostaza, vinagre, etc. 
21. Sal y condimentos

22. Café, chocolate, otras bebidas con cacao, té y otras hierbas aromáticas

23. Papas fritas, chitos, maicitos, patacones, besitos, etc.

24. Alimentos preparados fuera del hogar
25. Consumo por fuera del hogar

De las proporciones se agregaron las categorías 1 . Alimentos preparados fuera del hogar y 2. Consumo por fuera del hogar. La Tabla 1 presenta la información descriptiva de las variables dependientes.

Tabla 1. Información descriptiva de las variables dependientes analizadas, por proporción de gasto y grupos de alimentos (porcentaje).

\begin{tabular}{|c|c|c|c|c|c|}
\hline Proporción & Observaciones & Mínimo & Máximo & Promedio & $\begin{array}{c}\text { Desviación } \\
\text { Estándar }\end{array}$ \\
\hline $\begin{array}{l}\text { Consumo de alimentos por fuera del hogar y alimentos } \\
\text { preparados }\end{array}$ & 8644 & 0 & 100 & 62,32 & 37,86 \\
\hline Arroz, pastas alimenticias, avena, maíz, harinas y otros cereales & 8644 & 0 & 100 & 4,27 & 10,62 \\
\hline $\begin{array}{l}\text { Pescado de río, mar y otros productos marinos (frescos, } \\
\text { congelados y enlatados) }\end{array}$ & 8644 & 0 & 100 & 3,69 & 10,36 \\
\hline Aceites y grasas: aceite, manteca, mantequilla, margarina, etc. & 8644 & 0 & 100 & 3,44 & 8,14 \\
\hline Granos secos: fríjol, lenteja, garbanzo, etc. & 8644 & 0 & 100 & 2,28 & 6,32 \\
\hline Azúcar, panela y otros endulzantes & 8644 & 0 & 100 & 1,96 & 5,29 \\
\hline $\begin{array}{l}\text { Café, chocolate, otras bebidas con cacao, té y otras hierbas } \\
\text { aromáticas }\end{array}$ & 8644 & 0 & 100 & 1,95 & 5,33 \\
\hline Carne de res, cerdo, hueso y vísceras & 8644 & 0 & 100 & 1,56 & 6,51 \\
\hline Salsas, mayonesa, mostaza, vinagre, etc. & 8644 & 0 & 100 & 1,39 & 5,18 \\
\hline $\begin{array}{l}\text { Salchichas, jamón, mortadela, salchichón y otras carnes frías } \\
\text { preparadas }\end{array}$ & 8644 & 0 & 100 & 1,21 & 4,99 \\
\hline Sal y condimentos & 8644 & 0 & 100 & 1,15 & 4,32 \\
\hline Galletas de sal y de dulce & 8644 & 0 & 100 & 1,01 & 4,13 \\
\hline Pollo, otras aves y menudencias & 8644 & 0 & 100 & 0,99 & 4,60 \\
\hline Queso (animal y vegetal) & 8644 & 0 & 100 & 0,86 & 3,93 \\
\hline Frutas: manzanas, peras, naranjas, etc. & 8644 & 0 & 100 & 0,85 & 4,37 \\
\hline Leche (animal y vegetal) & 8644 & 0 & 100 & 0,83 & 3,83 \\
\hline Otros derivados de la leche: yogur, kumis, etc. & 8644 & 0 & 100 & 0,83 & 3,61 \\
\hline $\begin{array}{l}\text { Mermeladas, arequipe, bocadillos, compotas, dulces, helados, } \\
\text { etc. }\end{array}$ & 8644 & 0 & 100 & 0,81 & 3,49 \\
\hline Huevos & 8644 & 0 & 73 & 0,76 & 3,31 \\
\hline $\begin{array}{l}\text { Enlatados y encurtidos: arveja, fríjol, maíz, espárragos, } \\
\text { zanahoria, habichuela, etc. }\end{array}$ & 8644 & 0 & 100 & 0,67 & 3,36 \\
\hline Papas fritas, chitos, maicitos, patacones, besitos, etc. & 8644 & 0 & 100 & 0,57 & 2,71 \\
\hline $\begin{array}{l}\text { Verduras y hortalizas: tomate, cebolla, arveja, espinaca, apio, } \\
\text { zanahoria, etc. }\end{array}$ & 8644 & 0 & 100 & 0,56 & 3,12 \\
\hline Plátano, yuca, arracacha, ñame, papa y otros tubérculos & 8644 & 0 & 72 & 0,46 & 2,79 \\
\hline Pan, arepas, bollos y almojábanas & 8644 & 0 & 100 & 0,40 & 3,76 \\
\hline
\end{tabular}

Fuente: Elaboración propia, ENPH 2016-2017.

Se siguió una metodología cuantitativa que se divide en dos partes: análisis descriptivos y estimaciones del Clusters por enlace Ward, que es un método de agrupación jerárquico que busca la mínima varianza entre los datos para crear los grupos.

En la literatura, las diferencias socioeconómicas son operacionalizadas y analizadas por medio de variables como: edad, educación, ocupación, ingreso, sexo o región $n^{4-9,26-28}$. Para este artículo, se operacionalizaron como condiciones socioeconómicas ${ }^{29,30}$ las siguientes variables: presencia de al menos una mujer en el hogar (Si-1, No-0), el nivel educativo máximo del hogar (Ninguno-0, Primaria-1, Secundaria-2, Superior-3), la edad del jefe del hogar, la composición del hogar (Nuclear sin hijos-0, Nuclear con hijos-1, 
Monoparental-2, Unipersonal-3 y otras tipologías-4), la cantidad de personas en el hogar, material del piso de la vivienda del hogar (Tierra-0, Cemento-1, Madera-2, Mármol-3), el sector (Urbano-1, Rural-0) y la región (Bogotá D.C.-0, Atlántica-1, Central-2, Pacífico-3, Oriental-4, San Andrés-5 y Nuevos Departamentos-6).

\section{Resultados}

Entre los resultados principales, se encontró que más del $80 \%$ de los hogares colombianos consume alimentos por fuera del hogar o alimentos preparados (Tabla 2). El $62,42 \%$ del gasto del hogar destinado a alimentos es para productos preparados o para consumo de alimentos por fuera del hogar (Tabla 1).

Dentro de los alimentos a preparar, los productos más presentes en los hogares colombianos son: salsas y condimentos, aceites y grasas, pescados y similares, y granos o legumbres (Tabla 2). Mientras que entre los productos menos presentes en los hogares colombianos son: frutas, verduras, plátano y yuca (Tabla 2). Cuando se revisa la proporción de gasto, se encontró que los hogares gastan principalmente en arroz (4,27\%), pescado $(3,69 \%)$, aceite $(3,44)$, granos $(2,28 \%)$ y azúcar $(1,96 \%)$ (Tabla 1).

Tabla 2. Consumo de alimentos en los hogares colombianos - Presencia (porcentaje).

\begin{tabular}{lcc}
\hline Producto o servicio & Si & No \\
\hline Consumo de alimentos por fuera del hogar y alimentos preparados & 89,81 & 10,19 \\
Sal y condimentos & 32,70 & 67,30 \\
Aceites y grasas: aceite, manteca, mantequilla, margarina, etc. & 28,66 & 71,34 \\
Granos secos: fríjol, lenteja, garbanzo, etc. & 24,10 & 75,90 \\
Pescado de río, mar y otros productos marinos (frescos, congelados y enlatados) & 24,01 & 75,99 \\
Café, chocolate, otras bebidas con cacao, té y otras hierbas aromáticas & 23,40 & 76,60 \\
Salsas, mayonesa, mostaza, vinagre, etc. & 22,22 & 77,78 \\
Arroz, pastas alimenticias, avena, maíz, harinas y otros cereales & 21,74 & 78,26 \\
Azúcar, panela y otros endulzantes & 21,53 & 78,47 \\
Galletas de sal y de dulce & 19,60 & 80,40 \\
Salchichas, jamón, mortadela, salchichón y otras carnes frías preparadas & 15,64 & 84,36 \\
Mermeladas, arequipe, bocadillos, compotas, dulces, helados, etc. & 13,73 & 86,27 \\
Otros derivados de la leche: yogur, kumis, etc. & 13,44 & 86,56 \\
Queso (animal y vegetal) & 13,27 & 86,73 \\
Papas fritas, chitos, maicitos, patacones, besitos, etc. & 11,75 & 88,25 \\
Leche (animal y vegetal) & 10,43 & 89,57 \\
Enlatados y encurtidos: arveja, fríjol, maíz, espárragos, zanahoria, habichuela, etc. & 9,98 & 90,02 \\
Huevos & 9,76 & 90,24 \\
Carne de res, cerdo, hueso y vísceras & 9,41 & 90,59 \\
Frutas: manzanas, peras, naranjas, etc. & 9,12 & 90,88 \\
Pollo, otras aves y menudencias & 8,46 & 91,54 \\
Plátano, yuca, arracacha, ñame, papa y otros tubérculos & 5,97 & 94,03 \\
Verduras y hortalizas: tomate, cebolla, arveja, espinaca, apio, zanahoria, etc. & 5,81 & 94,19 \\
Pan, arepas, bollos y almojábanas & 5,36 & 94,64 \\
\hline
\end{tabular}

Fuente: Elaboración propia, a partir de la ENPH, 2016-2017.

Al clasificar el consumo de los hogares colombianos se encontró una solución óptima en la agrupación de los productos: 4 clusters o grupos (Figura 1). La Figura 1 muestra los clusters o grupos en los que se pueden clasificar los alimentos. La línea de color negro representa el corte a cuatro clusters. Analíticamente, los resultados se pueden dividir principalmente entre los hogares que gastan su presupuesto alimentario en consumo por fuera del hogar (grupo 4) y aquellos que gastan en alimentos para preparar (grupos 1, 2 y 3). La Tabla 3 presenta las medias de las proporciones de gasto por grupos $(1,2,3 \mathrm{y} 4)$. 
El grupo 4, el más grande de la muestra estudiada $(43,26 \%)$, es el que gasta principalmente en alimentos preparados y consumo de alimentos por fuera del hogar (Tabla 3). En promedio, los hogares del grupo 4 destinan un $88,61 \%$ del presupuesto alimenticio a dicho tipo de consumo. Éste se compone del porcentaje más alto de hogares sin presencia de mujeres en él. Se configura principalmente por hogares cuyo jefe del hogar tiene edades entre 30 y 40 años y el principal nivel educativo es medio. Sobre la estructura del hogar, se puede decir que este grupo se conforma mayoritariamente de la categoría Otras Tipologías, mientras que son menores las categorías nucleares con o sin hijos, monoparentales o unipersonales. Sus condiciones materiales de vida son altas, lo que corresponde a la variable material del piso baldosa, mármol, entre otras. Se ubican principalmente en el sector urbano, y en la región Atlántica.

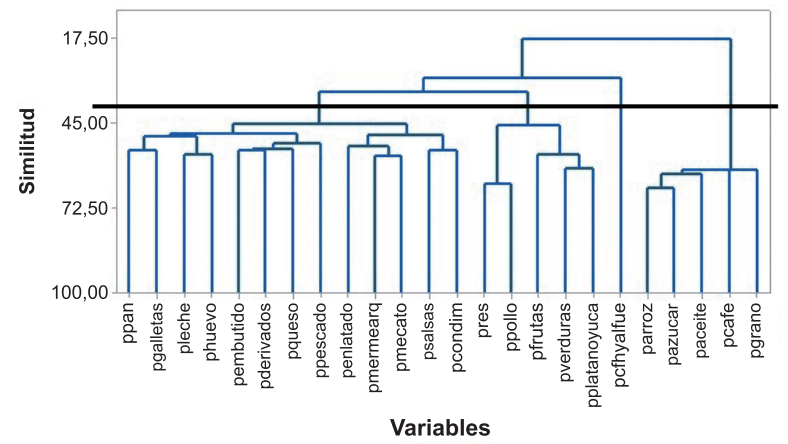

Figura. 1 Dendograma por productos (Enlace de Ward). Fuente: Elaboración propia, a partir de la ENPH, 2016-2017.

En contraste, el grupo 2, el más pequeño de la muestra $(2,94 \%)$, consume principalmente pescado (Tabla 3). De los grupos que gastan su presupuesto alimentario en alimentos para preparar, es el que tiene mayor porcentaje de hogares sin mujeres en él. Y se compone principalmente de hogares cuyos jefes de hogar tienen entre 45 y menos de 60 años. De los grupos que consumen alimentos para preparar, el grupo 2 es el que tiene el mayor porcentaje de hogares con condiciones materiales o de vida bajas, lo que corresponde a un mayor número de hogares con materiales de piso de tierra, cemento o gravilla.

Luego, el grupo $3(20,37 \%$ de la muestra) consume mayoritariamente arroz, aceite, grano, azúcar y café (Tabla 3), es el grupo que tiene mayor porcentaje de hogares con al menos una mujer en él. Se compone principalmente de hogares cuya edad del jefe del hogar es entre 30 y 44 años.

Y el grupo 1 (33,43\% de la muestra), que tiene la mayor proporción de gasto promedio en carne de res (Tabla 3), está conformado principalmente por hogares con niveles educativos medios; pero, entre grupos, es el que tiene más hogares con jefes con altos niveles educativos alcanzados. También, este grupo se compone principalmente de hogares con altos niveles con condiciones materiales, lo que significa que el porcentaje más alto de hogares tiene material del piso baldosa o mármol. Son hogares que pertenecen al sector urbano y a la región Central.

Tabla 3. Media de las proporciones de grupos de alimentos por grupos de alimentos y clusters (porcentaje).

\begin{tabular}{|c|c|c|c|c|}
\hline \multirow{2}{*}{ Productos } & \multicolumn{4}{|c|}{ Grupo } \\
\hline & 1 & 2 & 3 & 4 \\
\hline ppan & 0,84 & 0,31 & 0,44 & 0,05 \\
\hline pgalletas & 1,83 & 0,92 & 1,69 & 0,05 \\
\hline parroz & 0,73 & 0,34 & 19,72 & 0,01 \\
\hline pres & 2,92 & 0,32 & 2,82 & 0,01 \\
\hline ppollo & 1,69 & 0,23 & 2,01 & 0,01 \\
\hline pembutido & 2,33 & 0,76 & 1,89 & 0,05 \\
\hline ppescado & 4,82 & 49,78 & 2,99 & 0,02 \\
\hline pleche & 0,76 & 0,28 & 2,77 & 0,02 \\
\hline pqueso & 1,75 & 1,64 & 1,03 & 0,04 \\
\hline pderivados & 1,68 & 0,83 & 1,12 & 0,04 \\
\hline phuevo & 0,49 & 0,43 & 2,80 & 0,04 \\
\hline paceite & 5,55 & 0,06 & 7,72 & 0,02 \\
\hline pfrutas & 1,62 & 0,53 & 1,33 & 0,04 \\
\hline pverduras & 0,48 & 0,03 & 1,92 & 0,00 \\
\hline penlatado & 1,22 & 0,26 & 1,17 & 0,04 \\
\hline pgrano & 3,31 & 1,75 & 5,48 & 0,02 \\
\hline pplatanoyuca & 0,28 & 0,13 & 1,79 & 0,00 \\
\hline pazucar & 2,25 & 0,00 & 5,94 & 0,01 \\
\hline pmermearq & 1,73 & 0,91 & 0,91 & 0,04 \\
\hline psalsas & 2,63 & 0,97 & 2,29 & 0,03 \\
\hline pcondim & 2,08 & 0,82 & 1,80 & 0,14 \\
\hline pcafe & 2,95 & 0,11 & 4,67 & 0,02 \\
\hline pgolosinas & 1,21 & 0,99 & 0,61 & 0,03 \\
\hline
\end{tabular}

Fuente: Elaboración propia, a partir de la ENPH, 2016-2017.

\section{Discusión}

El estudio de la relación entre proporciones de gasto en alimentos de los hogares y sus diferencias socioeconómicas ayuda a revelar patrones de consumo. Respecto a los patrones de consumo, si hay algo que pudiera denominarse como una "cocina nacional", en Colombia puede decirse que se compone de: sal y condimentos, aceite y grasas, granos, pescado, salsas, arroz y azúcar. 
Respecto al gasto en alimentos para preparar, puede afirmarse que los grupos socioeconómicos más altos consumen res, pollo, frutas y verduras. Pero realmente el promedio de la proporción del gasto en frutas y verduras es bajo ${ }^{27}$. Mientras que los grupos socioeconómicos medios y bajos consumen principalmente pescado, embutidos, aceites y grasas, y una amplia gama de productos procesados. Especialmente el resultado sobre el pescado llama la atención, debido que es contrario a lo que se ha reportado en la literatura: a mayores niveles socioeconómicos mayor consumo de pescado ${ }^{1,8,23,31}$.

Ahora bien, el porcentaje de hogares que comen alimentos por fuera del hogar es bastante alto, lo que es correspondiente con los estudios de mercado sobre el sector de servicios de alimentos ${ }^{32}$, por lo que los hogares que lo componen son bastante disímiles. Pero si se pudiera hablar de un patrón de consumo, a este grupo, también pertenecen principalmente los hogares de las zonas urbanas.

Ahora bien, dado que Colombia es un país joven, en este grupo es explicable que esté mayoritariamente conformado por personas con edades entre 30 y 44 años, que se consideran de la población económicamente activa $y$ en edad de estudiar, los que seguramente tendrán mayores incentivos para tener un consumo por fuera del hogar ${ }^{33}$. Y quienes consumen fuera del hogar tienden a adquirir menos alimentos para preparar. Luego, es razonable que sean bajas las proporciones de gasto que destinan a la gama de productos presentados. Finalmente, vale la pena resaltar que, en similitud, en línea con la literatura realizada en otras latitudes y presentada al inicio de este documento, se pueden afirmar desigualdades en el consumo de alimentos del hogar en Colombia por: género, ciclo de vida, nivel educativo o condiciones materiales ${ }^{27}$. Sin embargo, es importante seguir revisando a profundidad de manera desagregada los productos alimentarios. Pues en la desagregación de los productos se pueden encontrar diferencias significativas con la literatura existente y realizada para otros países. En especial para los patrones de consumo de carnes, frutas, verduras y pescado ${ }^{1,5,6,9,27,36,37}$.

\section{Conclusiones}

Los resultados demuestran que cuando se habla de consumo de alimentos en los hogares es necesario tener en cuenta las condiciones socioeconómicas de los hogares y una visión más amplia contextual para comprender los patrones de consumo de los hogares. Porque si sabemos que el sobrepeso y la obesidad está en aumento en Colombia ${ }^{34}$; y si se desea incidir en hábitos de consumo de alimentos saludables, es fundamental tener presente que:

1. Dada la alta proporción de hogares que consumen fuera del hogar, debe revisarse a profundidad la calidad nutricional de los bienes y servicios ofrecidos en el mercado del consumo de alimentos por fuera del hogar.

2. En general, en el territorio colombiano, es bajo el consumo de frutas y verduras; y es alto el consumo de grasas, carnes y azúcares.

3. Es necesario revisar cómo incidir en un cambio a una dieta más rica en nutrientes y saludable, pero de acuerdo con las posibilidades, tradiciones $\mathrm{y}$ necesidades de los hogares, en especial cuando se ha comprobado que el cambio de las prácticas alimentarias en los hogares es lento ${ }^{35}$.

\section{Agradecimientos}

Este balance forma parte del proyecto "¿Qué vamos a comer? Estudio de la relación entre condiciones socioeconómicas y consumo de alimentos en Colombia, 1991-2017". La realización fue posible gracias a la Comisión Nacional de Investigación Científica y Tecnológica: CONICYT-PCHA/Beca Doctorado Nacional/2017-21170258 y al Instituto de Sociología de la Pontificia Universidad Católica de Chile. Expreso mi gratitud a Séverine Gojard y Marie Plessz del Centre Maurice Halbwachs (Centre National de la Recherche Scientifique, École Normale Supérieure, École des Hautes Études en Sciences Sociales) por los diálogos y textos compartidos para el desarrollo de este trabajo. También estoy muy agradecida con los evaluadores doble ciego por sus valiosos comentarios que contribuyeron a mejorar la versión final de este artículo.

\section{Conflicto de interés}

La autora declara no tener conflictos de interés reales o potenciales sobre el trabajo desarrollado o los resultados alcanzados.

\section{Referencias}

1. Bourdieu P. La Distinción. Madrid: Grupo Santillana de Ediciones; 1998.

2. Grignon C, Grignon C. Styles d'alimentation et goûts populaires. Rev Française Sociolog. 1980; 21(4): 531-569. doi: https://doi.org/10.2307/3320833

3. Grignon C, Grignon C. Alimentation et stratification 
social. Cahiers Nutrition et Diététique, 1981; 16: 207-217.

4. Darmon N, Drewnowski A. Does social class predict diet quality?. Am J Clin Nutrition. 2008; 87(5): 1107-1117. doi: 10.1093/ajcn/87.5.1107

5. Sánchez-Villegas A, Martínez JA, Prättäla R, Toledo E, Roos G, Martínez-González MA. A systematic review of socioeconomic differences in food habits in Europe: Consumption of cheese and milk. Eur J Clin Nutr. 2003; 57: 917-929. doi: 10.1038/ sj.ejen. 1601626

6. Andrieu E, Darmon N, Drewnowski A. Low-cost diets: more energy, fewer nutrients. Eur J Clin Nutr. 2006; 60: 434-436. doi: 10.1038/sj.ejen.1602331

7. De Irala-Estévez J, Groth M, Johansson L, Oltersdorf U, Prättäla R, Martínez-González, MA. A systematic review of socioeconomic differences in food habits in Europe: Consumption of fruit and vegetables. Eur J Clin Nutr. 2000; 54: 706-714. doi: https://doi.org/10.1038/sj.ejcn.1601080

8. Drewnoski A, Monsivais P, Maillot M, Darmon N. Low-energy-density diets are associated with higher diet quality and higher diet costs in French adults. J Am Diet Assoc. 2007; 107(6): 1028-1032. doi: 10.1016/j.jada.2007.03.013

9. López-Azpiazu I, Sánchez-Villegas A, Johansson L, Petkeviciene J, Prättäla R, Martínez-González MA. Disparities in food habits in Europe: systematic review of educational and occupational differences in the intake of fat. J Hum Nutr Diet. 2003; 16(5): 349-364. doi: 10.1046/j.1365-277x.2003.00466.x.

10. Harris M. Bueno para comer. Madrid: Alianza Editorial; 2009.

11. Steiner Ph. La sociologie économique. Paris: La Découvert; 2011.

12. Reckwitz A. Toward a theory of social practices: A development in culturalist theorizing. Eur J Soc Theo. 2002; 5(2): 243-263. doi: https://doi. org/10.1177/13684310222225432

13. Schatzki TR, Knorr Cetina K, von Savignay E. (Editores.) The practice turn in contemporary theory. London: Routledge; 2001.

14. Weber, M. Economía y Sociedad: Esbozo de sociología comprensiva. España: Fondo de Cultura Económica; 2002.

15. Dubuisson-Quellier S. Le prestataire, le client et le consommateur. Sociologie d'une relation marchande. RevFrançaise Sociolog. 1999; 40(4): 671-688. doi: 10.2307/3322854

16. Poulain JP. Sociologies de l'alimentation: Les mangeurs et l'espace social alimentaire. Paris: Presses Universitaires de France; 2002.

17. Régnier F, Lhuissier A, Gojard S. Sociologie de l'alimentation. Paris: La Découverte; 2006.

18. Kamphuis C, Jansen T, Mackenbach J, van Lenthe F. Bourdieu's Cultural Capital in Relation to Food Choices: A Systematic Review of Cultural Capital Indicators and an Empirical Proof of Concept. PLoS ONE, 2015; 10(8): 1-19. doi: https://doi. org/10.1371/journal.pone.0130695

19. Wills, W., Backett, K., Roberts, M-L., \& Lawton, J. The framing of social class distinctions through family foods and eating practices. Socio Rev. 2011; 59(4): 725-740. doi: https://doi.org/10.1111/j.1467954X.2011.02035.x

20. Beagan B, Chapman G, Power, E. Cultural and symbolic capital with and without economic constraint: Food shopping in low-income and highincome Canadian families. Food, Cult \& Soc. 2016; 19(1): 45-70. doi: 10.1080/15528014.2016.1145002

21. Baumann S, Szabo M, Johnston, J. Understanding the food preferences of people of low socioeconomic status. J Consu Cult. 2017; 1-24. doi: 10.1177/1469540517717780.

22. De Saint Pol T. Les évolutions de l'alimentation et de sa sociologie au regard des inégalités sociales. L'Année sociolog. 2017; 67(1): 11-22. doi: 10.3917/ anso. 171.0011

23. Van Lenthe F, Jansen T, Kamphuis C. Understanding socio-economic inequalities in food choice behaviour: can Maslow's pyramid help? Brit J Nutr. 2015; 113: 1139-1147. doi: https://doi.org/10.1017/ S0007114515000288

24. Bertollo M, Martire Y, Rovirosa A, Zapata M.E. Patrones de consumo de alimentos y bebidas según los ingresos del hogar de acuerdo a los datos de Encuesta Nacional de Gastos de los Hogares (ENGHo) del año 2012-213. Diaeta, 2015; 33(153): 7-18.

25. Garza-Montoya BG, Ramos-Tovar ME. Cambios en los patrones de gasto en alimentos y bebidas de hogares mexicanos (1984-2014). Sal Púb Mex. 2017; 59(6): 612-620. doi: 10.21149/8220

26. Rodríguez E, Berges M, Casellas K. Diferencias regionales en el consumo de alimentos de los hogares argentinos. Rev Arg Eco Agra. 2001; 4(1): 3-12.

27. Torres Pabon G. ¿En qué gastamos para comer y cuánto?: condiciones socioeconómicas y presupuesto para el consumo de alimentos. Rev Col Sociolo. 2019; 42(2): 191-228. doi: https://doi. org/10.15446/rcs.v42n2.74809

28. Zapata, M.E., Rovirosa, A. \& Carmuega, E. Cambios en el patrón de consumo de alimentos y bebidas en Argentina, 1996-2013. Sal Colec. 2016; 12(4): 473486. doi: https://doi.org/10.18294/sc.2016.936 
29. Galobardes B, Shaw M, Lawlor D, Lynch J, Smith, G.D. Indicators of socio-economic position (Part 1). J EpiComm Heal. 2006; 60: 7-12. doi: https://doi. org/10.1136/jech.2004.023531

30. Galobardes B, Shaw M, Lawlor D, Lynch J, Smith, G.D. Indicators of socio-economic position (Part 2). J EpiComm Heal. 2006; 60: 7-12. doi: https://doi. org/10.1136/jech.2004.023531

31. Lallukka T, Laaksonen M, Rahkonen O, Roos E, Lahelma E. Multiple socio-economic circumstances and healthy food habits. Eur JClin Nutr. 2007; 61: 701-710. doi: https://doi.org/10.1038/ sj.ejcn. 1602583

32. Euromonitor International. Consumer Foodservice in Colombia: Country report (en línea). www. euromonitor.com, último acceso 20 de abril de 2018.

33. Van den Eeckhout P. The history of labour and labour relations in hotel and restaurants in Western Europe and the United Stated in the 19th and 20th centuries. Food \& Hist. 2013; 11(2): 199-377. doi: https://doi.org/10.1484/J.FOOD.5.102110

34. Encuesta Nacional de la Situación Nutricional. Jóvenes y Adultos 18 a 64 años. https://www.icbf. gov.co/sites/default/files/portada-ensin6.jpg

35. Warde A. Consumption, food and taste: Culinary antinomies and commodity culture. London: SAGE Publications; 1997.

36. Herrán O, Patiño G, Zea MP. Patrones de consumo alimentario y pobreza monetaria en Colombia. Rev Chil Nutr. 2018; 4: 372-380. doi: https://doi. org/10.4067/S0717-75182018000500372

37. Herrán O, Patiño GA, Del Castillo SE. La transición alimentaria y el exceso de peso en adultos evaluados con base en la Encuesta de la Salud Nutricional en Colombia, 2010. Biomédica, 2016, 36: 109-120. doi: https://doi.org/10.7705/biomedica.v36i1.2579 